\title{
LV SCADA PROJECT: IN-FIELD VALIDATION OF A DISTRIBUTION STATE ESTIMATION TOOL FOR LV NETWORKS
}

\author{
Pedro BARBEIRO \\ INESC TEC - Portugal \\ pedro.p.barbeiro@inesctec.pt
}

Luís SECA

INESC TEC - Portugal

luis.seca@inesctec.pt

Francisco MELO

EDP DISTRIBUIÇÃO - Portugal

francisco.melo@edp.pt

\begin{abstract}
The LV SCADA project aimed at the development of advanced technical, commercial and regulatory solutions to contribute for an effective smart grid implementation. One of the biggest challenges of the project was related with the lack of characterization that usually exists in $L V$ networks, together with the almost non-existing observability. In order to overcome these issues, a LV management system integrating a state estimation tool based on artificial intelligence techniques was developed. The tool is currently installed in one pilot demonstration site that aggregates $2 \mathrm{MV/LV}$ substations. In this paper the performance of tool in real environment is evaluated and the results gathered from the pilot site are analyzed.
\end{abstract}

\section{INTRODUCTION}

The growing integration of electric vehicles, microgeneration and storage units, as well as the possibility of having demand side management solutions are contributing to numerous challenges in the nowadays' Low Voltage (LV) systems. In order to enable the safer integration of all the assets in smart grids, monitoring the network in quasi-real-time is a compulsory requisite.

Smart Meters (SM) may be one of the cornerstones for Distribution System Operators (DSO) in order to have an effective real-time monitoring of their LV grids. As the market for these devices continues to explode, several options are already available today. Some of these solutions are able to remotely monitor and transfer electrical information in real-time, while the others, although can measure and store locally electrical information for billing purposes, cannot guarantee realtime access to information. SM with the capability of transmitting real-time measurements $\left(\mathrm{SM}_{\mathrm{RT}}\right)$ will contribute decisively to increase the observability level in LV grids, but even thinking that these solutions are becoming more cost-effective, monitoring all grid points in real-time will be for sure economically unfeasible. Therefore, the existence of an accurate Distribution State Estimation (DSE) module as part of a new generation of LV management systems will be indispensable for properly monitor, control and operate LV systems.

A big issue usually faced in LV networks is related to the lack of knowledge that DSOs have about their real status, i.e., grids topology and electrical characteristics are often unknown. This fact makes the classical state estimation techniques not suitable to be used. In order to tackle these limitations, in [1] the authors proposed an alternatively approach based on a particular type of neural networks the Autoencoders. This new approach accounts for very promising results in terms of estimation accuracy and computational times [1]. Although data from real networks was used in these studies, measurements from SM were artificially generated and the algorithm was only validated through simulation. An in-field validation is therefore required in order to assess the effectiveness of such tool when in presence of real-world constraints.

The LV SCADA project, started in September 2012 and finished in June 2015, was a project led individually by Efacec Automation and supported by the Portuguese DSO and a R\&D Institution (INESC TEC) aiming the development of an advanced system that contributes for an effective smart grid implementation, creating flexible solutions to allow the anticipation of future trends, supporting the introduction of new technical, commercial and regulatory solutions to improve network operation efficiency. The main tasks of the project included the development of advanced processing modules, consumption and active production control algorithms, LV fault management, intelligent alarm processing, and also interfaces to other utility corporate systems [2].

One of main tools supporting the set of new modules developed in the project was a Distribution State Estimator (DSE) for LV networks based on the model proposed in [1]. The tool is currently integrated and being tested in 2 LV networks explored by the Portuguese DSO - EDP Distribuição. In this paper the performance of the DSE tool in a real environment is evaluated using data gathered from these real systems. Estimation accuracy and running times are the two indicators analyzed. Different scenarios regarding the number and location of the $\mathrm{SM}_{\mathrm{RT}}$ installed in field are analyzed for comparison purposes. A brief description of the Graphical User Interface (GUI) developed within the project is also provided.

\section{THE DSE TOOL FOR LV NETWORKS}

In the scope of the LV SCADA project, the DSE tool proposed is a real-time functionality that was designed not only to provide a complete estimation of voltage magnitudes, but also the injected active power values in a given bus, if required. In order to meet this purpose the DSE tool takes benefit of the set of new sensors that are being installed in LV grids: SM and advanced RTUs such as Distribution Transformer Controllers (DTC) that are installed at secondary substation level, constituting an 


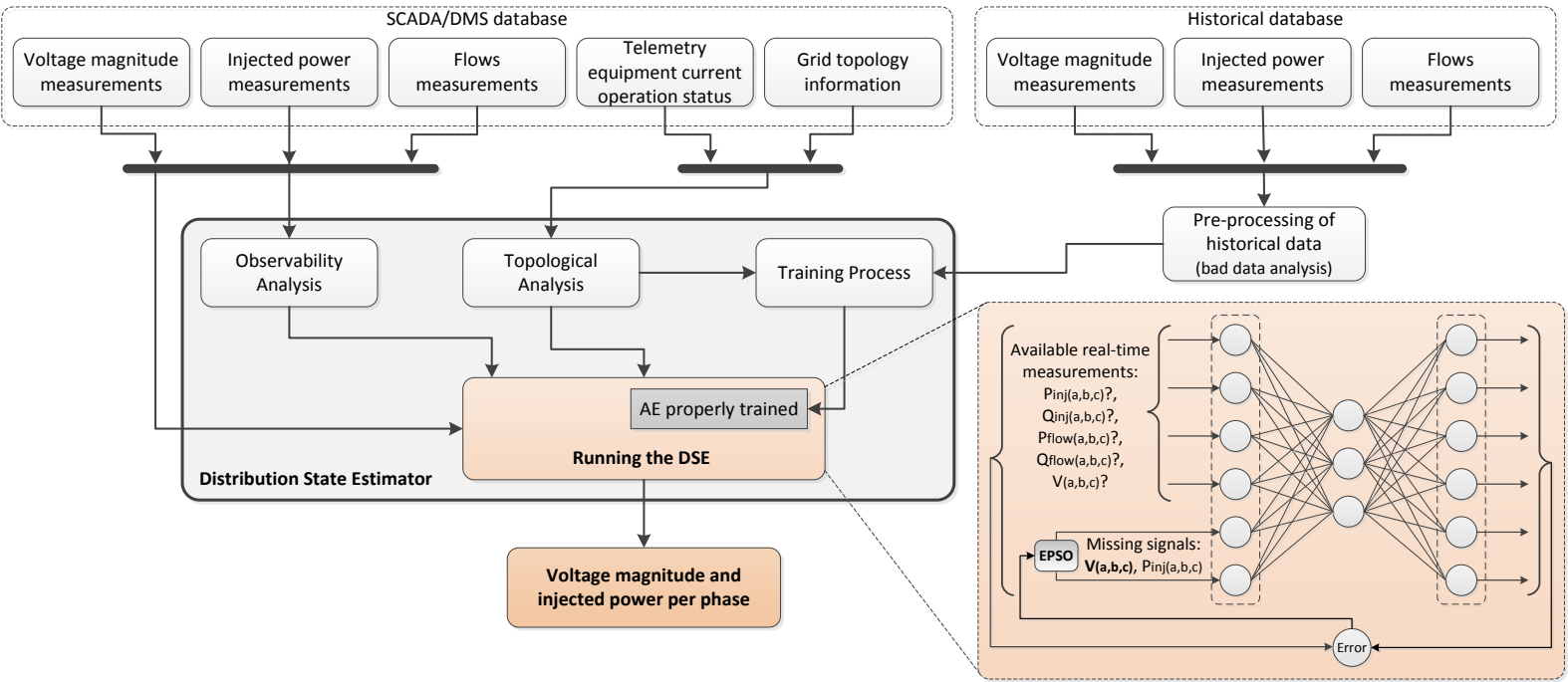

Figure 1: Functional diagram of the DSE tool.

Advanced Metering Infrastructure (AMI). The AMI is regarded as the source of information for elementary SCADA modules such as the Data Acquisition and Information Processing module which is transversal to all the system modules. In this module, there are two important sources of data that need to be kept up-to-date in order to feed the DSE tool properly: the SCADA/DMS database and the historical database. The first contains information required for the DSE online execution, while the second stores information which is used for training the DSE offline. Figure 1 is an overview of the functional diagram of the DSE tool, where it can be seen the interaction between the different blocks.

\section{Algorithm}

As stated, the "core" of the algorithm supporting the DSE tool is based on the concept of an AE that, after being properly trained, can be used within an optimization process to provide values for the missing signals, i.e., the system state variables to be estimated. An Evolutionary Particle Swarm Optimization (EPSO) was the algorithm chosen for reconstructing the state variables. The running process of the DSE may be better understood observing the scheme at the bottom right side of Figure 1. A detailed description of training and running processes, including the control parameters involved can be found in [1].

In the context of the LV SCADA project, the DSE can be configured for running in three distinct modes: manual, supervised or automatic. In the manual mode, the DSE only runs on user request (by pressing the "Execute now" button - see Figure 2), whereas in the other two modes it run automatically according with a configurable time step.

\section{Graphical User Interface}

In order to enable the interaction between the functionalities developed in the LV SCADA project and the user, a simple and intuitive GUI was developed. Figure 2 is an assembly of several illustrative screenshots taken from the GUI where the main features related with the
DSE tool were marked with numbers. The background corresponds to the main window usually presented to the user (numbers 1. to 4.) and the two small windows pasted on it (numbers 5. and 6.) are related with options that are usually accessed in separated windows. Below each one of these features is briefly described:

1. List containing all system voltages values per phase, date of its acquisition/estimation and the correspondent equipment/location. In the field "Telemetered", real-time values are marked with word "Yes" and estimated values with word "No"

2. Shows the equipment which voltage values are currently out of the defined limits.

3. List specifying the type of all alarms occurred in the system (e.g. voltage violations).

4. Description of all actions taken either manually by the user or autonomously by the system

5. Diagram containing all the network equipment plotted on a georeferenced map. The connection between equipment may be only schematics, i.e., without electrical meaning (when topology is unknown but GPS coordinates are known) or electrically representative if grid topology is known.

6. In this window, the user can run the training process, define the thresholds for voltage violations, the time step between runs and configure the tool's operation mode, among other control parameters.

\section{In-field Validation of the Tool}

The DSE is being tested in a demonstrator that aggregates $2 \mathrm{MV} / \mathrm{LV}$ substations, each one feeding a LV network with several single-phase and three-phase clients, as well as micro-generation units (PV panels). PLC Prime communications are used to exchange data between the $\mathrm{SM}$ and the DTC deployed in the correspondent MV/LV substation. The DTC aggregates and computes data from the existing sensors in the substation and the ones distributed among the LV feeders (SM at customers' premises). All SM are equipped with technology suitable 


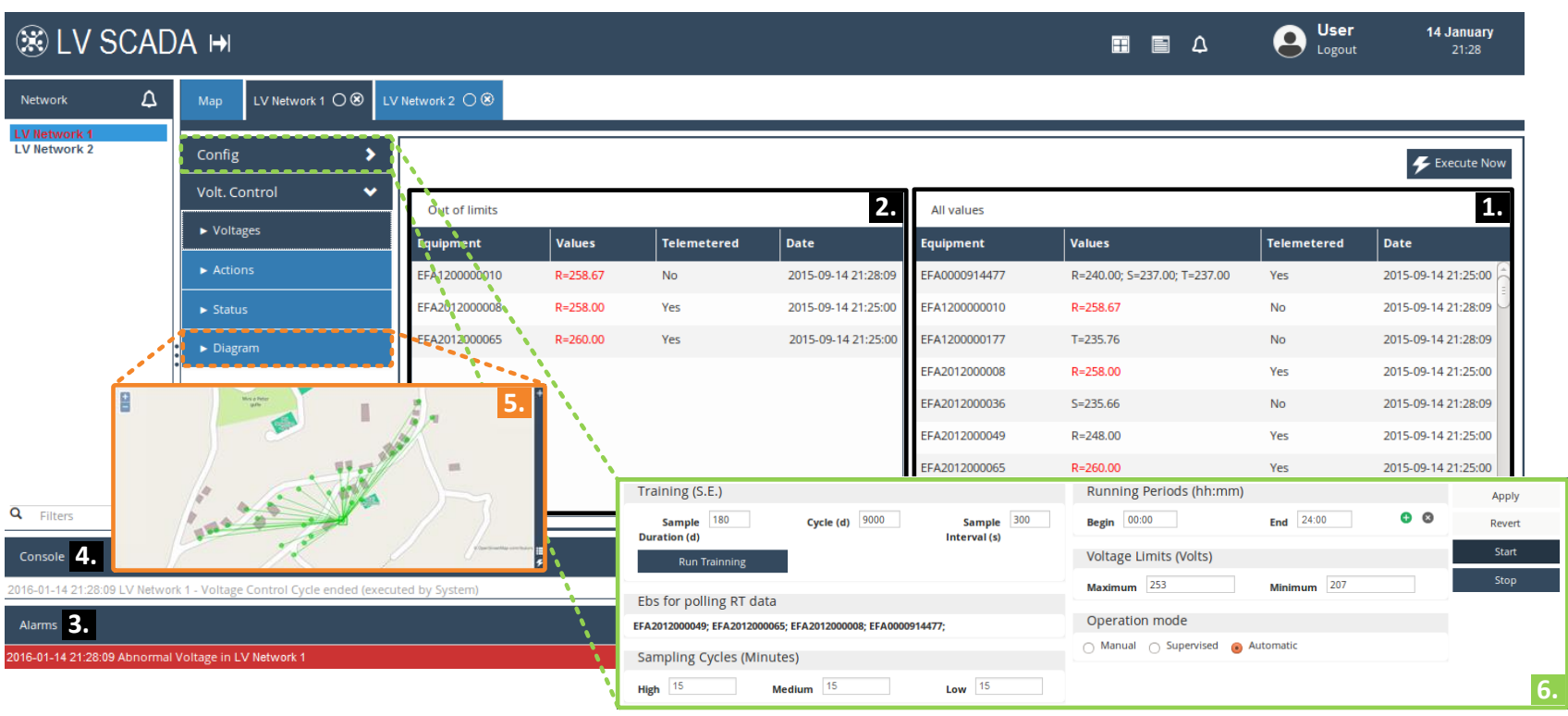

Figure 2: Overview of the GUI developed within the LV SCADA project

to transmitting near real-time information (average values from the last 15 minutes) to the DTC, thus the $\mathrm{SM}_{\mathrm{RT}}$ that are providing data to the DSE algorithm can be easily varied. Nevertheless, in order to properly evaluate the DSE tool, only a limited number of SM is configured as $\mathrm{SM}_{\mathrm{RT}}$. The remaining ones are periodically accessed on request (e.g. once a day) in order to gather historical data records that can be used to train the DSE.

Once it is possible to obtain real historical data records from all SM existing in the field, the evaluation of the DSE tool accuracy can be done offline through the comparison between such records and the correspondent estimated values. In this sense, data of the last available month was gathered from the demonstrator and is used to evaluate the tool performance. Two months of historical data prior to this month was used for training the DSE.

\section{RESULTS AND ANALYSIS}

The results that allow evaluating the DSE tool accuracy and running times are presented in this section. Due to space restrictions, only results for the estimation of voltage magnitude values in one of the two LV grids available are shown. Since a bigger system is likely to be more challenging to perform state estimation, the network with the largest number of customers for whom reliable historical data were available was selected (43 clients). Regarding the number and location of $\mathrm{SM}_{\mathrm{RT}}$ four scenarios are analyzed. These scenarios are characterized in Table I, in terms of the number of $\mathrm{SM}_{\mathrm{RT}}$ and the percentage that it represents in the universe of the available SM. It is important to state that in scenarios 1, 2 and 4, the $\mathrm{SM}_{\mathrm{RT}}$ were chosen after a correlation analysis between the electrical quantities available (historical data was used in this process). The most electrically representative SM and less correlated to each other were firstly chosen until reaching the number of $\mathrm{SM}_{\mathrm{RT}}$ desired in each scenario. Differently, in scenario 3, a random selection was applied.
Table I: Characterization of the $S M_{R T}$ scenarios

\begin{tabular}{cccc}
\hline Scenario 1 & Scenario 2 & Scenario 3 & Scenario 4 \\
\hline $6(14 \%)$ & $12(28 \%)$ & $12(28 \%)$ & $18(42 \%)$ \\
\hline
\end{tabular}

\section{Accuracy assessment}

In Figure 3, relative frequency histograms with the voltage magnitude absolute errors (calculated as the difference between the estimated and the measured values) computed in intervals of $1 \mathrm{~V}$ are presented for each analyzed scenario. The results include errors for all the estimated quantities and for all the 2280 times instants analyzed (1 month). The red line located around $5 \mathrm{~V}$ represents a threshold of about $2 \%$ of the nominal voltage $\left(U_{n}\right)$, a value assumed as an acceptable limit for a "good" estimation. Comparing the four scenarios, it is clear that, when the number of $\mathrm{SM}_{\mathrm{RT}}$ is increased, the estimation error tends to be distributed in the lower error ranges. This result is particularly notorious in the range 1 to $2 \mathrm{~V}$, where the relative frequency has increased from $49.8 \%$ in scenario 1 to $71.5 \%$ in scenario 2 . Such fact can also be verified observing the values distributed above the $2 \%$ limit, where the frequency of this errors became lower with the increase number of $\mathrm{SM}_{\mathrm{RT}}$ (with less expression since the rate of errors in this range is already very low in all scenarios). These results demonstrate the good accuracy of

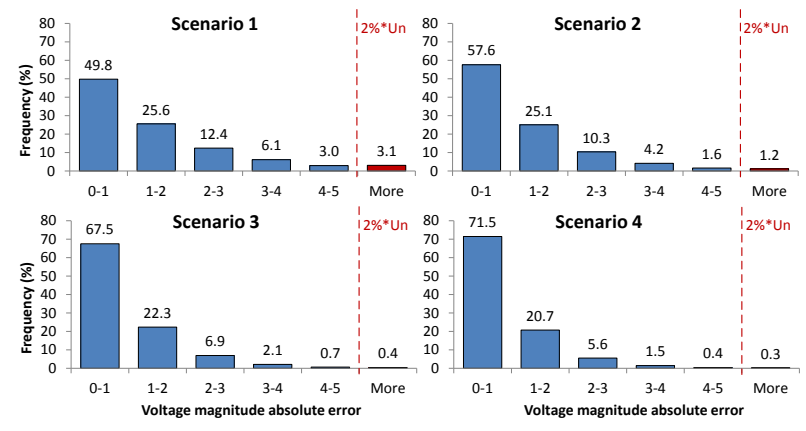

Figure 3: Relative frequency histograms 
the DSE tool regarding the estimation of voltage values with real data gathered from the field, even for a scenario with only $14 \%$ of $\mathrm{SM}_{\mathrm{RT}}$ (scenario 1). In Table II are computed different errors indicators for all the scenarios analyzed and considering the entire evaluation set under analysis. The Mean Absolute Error (MAE) was calculated according with equation (1).

$$
M A E=\frac{1}{N \times T} \sum_{n=1}^{N} \sum_{t=1}^{T}\left|R_{t n}-E_{t n}\right|
$$

where $R$ is the real measurement $E$ is the estimated value, $\mathrm{T}$ is the total number of time instants evaluated and $\mathrm{N}$ is the total number of estimated values.

The results depicted in Table II and Figure 3 highlights the importance of use methodologies for finding the most suitable locations to place a $\mathrm{SM}_{\mathrm{RT}}$. As it can be seen, by comparing scenario 2 and 3 , scenario 3 accounts for better results, despite both have the same numbers of $\mathrm{SM}_{\mathrm{RT}}$. In order to have a perspective of the error distribution among the grid and the perception of how the $\mathrm{SM}_{\mathrm{RT}}$ distribution may affect accuracy, the MAE for each customer in the scenarios analyzed is computed in Figure 4 by means of a color gradient. The dark red stands for the highest MAE, while the dark blue stands for the lowest. The $\mathrm{SM}_{\mathrm{RT}}$ are represented as white boxes in the figure.

As it can be seen in Figure 4, the highest MAE tends to be distributed in areas electrically distant from nodes with a $\mathrm{SM}_{\mathrm{RT}}$. Once more, it is evident the improving on the estimation error from the scenario 1 to scenario 4 .

\section{Running times}

In order to assess the total time associated with the DSE process, it is necessary to consider the running time of the
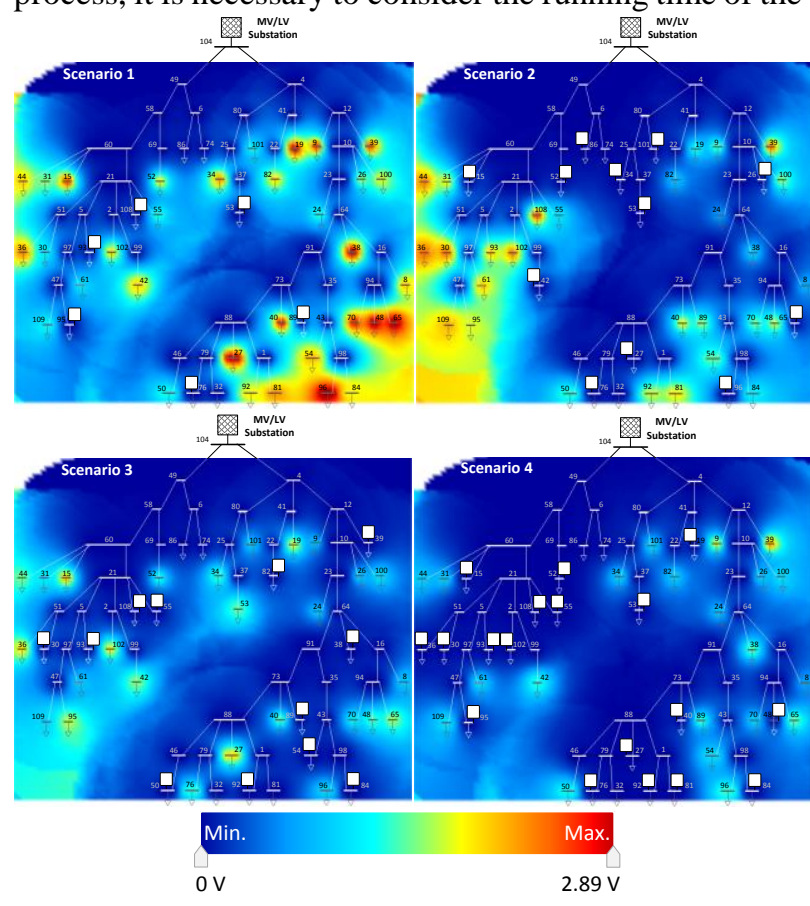

Figure 4: Voltage magnitude MAE distribution
Table II: Error indicators

\begin{tabular}{ccccc}
\hline & Scenario & Scenario & Scenario & Scenario \\
& $\mathbf{1}$ & $\mathbf{2}$ & $\mathbf{3}$ & $\mathbf{4}$ \\
\hline Max. & 15.77 & 10.82 & 10.53 & 8.56 \\
Min. & 0.00 & 0.00 & 0.00 & 0.00 \\
MAE & 1.44 & 1.15 & 0.90 & 0.81 \\
\hline
\end{tabular}

algorithm itself, as well as the time spent on acquisition and synchronization of the measurements gathered from the $\mathrm{SM}_{\mathrm{RT}}$ installed in the field. In the network under analysis, the system takes about 2 minutes in average to communicate with all the $\mathrm{SM}_{\mathrm{RT}}$ in the scenario with more $\mathrm{SM}_{\mathrm{RT}}$ and less than 1 second to finish the entire state estimation process (in a computer equipped with an IntelCore i7 based processor). Nevertheless, tests carried out in field have demonstrated that, instead PLC if GPRS technology is used to communicate with the $\mathrm{SM}_{\mathrm{RT}}$, the total time can be reduced to around 30 seconds in average.

\section{CONCLUSION}

In this paper a DSE tool developed in the context of LV SCADA project was evaluated through the analysis of recent data gathered from a pilot demonstration site. The results demonstrated that the DSE tool allows the estimation of voltage magnitude values with a significant high accuracy degree, even in a real environment. The tool proved to be apt for dealing with real world constrains, such as the diversity of load and generation profiles, noisy measurements, real-time communications, data synchronization, etc. Regarding the time performance, it was verified that the total time needed to complete the state estimation process is influenced by communication time between devices rather than the running time of the algorithm itself, which may be neglected. The total running times are satisfactory for accomplish the main goals intended, i.e., at a first stage, this DSE tool is likely to be oriented to the identification of locations where voltage violations frequently occurs, either due to high generation or high load demand. In these cases, the DSE tool can be used within a voltage control algorithm to test the efficacy of different control solutions.

The DSE presented proved to be an interesting option for future LV management systems, particularly for poorly characterized networks, as it allows monitoring them in an effective and straightforward manner.

\section{REFERENCES}

[1] P. N. P. Barbeiro, H. Teixeira, J. Krstulovic, J. Pereira, and F. J. Soares, 2015, "Exploiting autoencoders for three-phase state estimation in unbalanced distributions grids", Electric Power Systems Research, vol. 123, 108-118.

[2] N. Silva, P. Silva, L. Seca, A. Madureira, J. Pereira, F. Melo, 2015, "LV SCADA - How to Effectively Manage LV Networks with Limited Topology and Electrical Characteristics Data”, in CIRED 2015. 